\title{
Nose tip refinement using interdomal suture in caucasian nose
}

\author{
Rogério Pasinato', Marcos Mocelin², Cezar Augusto Sarraf Berger³.
}

1) Master. Associate Professor, Department of Otorhinolaryngology, Federal University of Paraná (UFPR). Doctor's ENT Hospital IPO.

2) Doctor. Professor, Department of Otorhinolaryngology, Federal University of Paraná (UFPR). Otolaryngologist Hospital IPO.

3) Master in Surgery. Volunteer lecturer, Department of Otolaryngology, Federal University of Paraná (UFPR). Otolaryngologist Hospital IPO.

Institution: Hospital de Clinicas da Universidade Federal do Paraná (HC-UFPR) / Hospital IPO. Curitiba / PR - Brazil.

Mailing address: Rogério Pasinato - Avenida República Argentina, 2069-Bairro Água Verde -Curitiba / PR - Brazil - Zip code: 80620-010 - E-mail: rogpasinato@ hotmail.com Article received in October 31, 2011. Article approved in February 6, 2012.

\section{SUMMARY}

Introduction: Refinement of the nose tip can be accomplished by a variety of techniques, but currently, the use of sutures in the nasal tip with conservative resection of the alar cartilage is the most frequently recommended approach.

Objective: To classify the nasal tip and to demonstrate the interdomal suture applied to nasal tip refinement in the Caucasian nose, as well as to provide a simple and practical presentation of the surgical steps.

Method: Development of surgical algorithm for nasal tip surgery: 1. Interdomal suture (double binding suture), 2. Interdomal suture with alar cartilage weakening (cross-hatching), 3. Interdomal suture with cephalic removal of the alar cartilage (McIndoe technique) based on the nasal tip type classification. This classification assesses the interdomal distance (angle of domal divergence and intercrural distance), domal arch width, cartilage consistency, and skin type. Interdomal suture is performed through endonasal rhinoplasty by basic technique without delivery (Converse-Diamond technique) under local anesthesia. Conclusion: This classification is simple and facilitates the approach of surgical treatment of the nasal tip through interdomal suture, systematizing and standardizing surgical maneuvers for better refinement of the Caucasian nose.

Keywords: rhinoplasty, suture techniques, nose.

\section{INTRODUCTION}

Type of work: Surgical technique.

Nasal tip surgery represents a challenge to rhinoplasty and requires the knowledge of essential anatomy and aesthetic points (Figure 1). Several techniques can be performed to improve nasal tip definition, such as cartilaginous resection, tip grafts, or suture placement. Resection techniques have gradually been replaced by maneuvers that are effective in preserving more of the nasal tip support mechanisms (1-10). Education on suture techniques on the nasal tip through external access combined with surgeon sensibility has enabled great progress in the applicability of closed-access techniques. The surgical algorithm is fundamental to the comprehension, judgment, and performing of surgical maneuvers, being necessary to surgery systematization whether at the clinic or at educational institutions.

The objective to classify the approach to nasal tip refinement through septocolumellar and intercartilaginous bilateral incisions based on nasal tip type (classification proposed by the authors). This classification considers the interdomal distance (angle of domal divergence and intercrural distance), domal arch width, alar cartilage consistency, and skin type (Figures 2 and 3). To develop a surgical algorithm based on the findings that describes the proposed maneuvers.

\section{METHOD}

The nasal tip type evaluation must be closely held. Skin inspection, lateral inferior lower lateral cartilage palpation, and measurement of angles and distances are fundamental to define the nasal tip type of the Caucasian nose (Table 1). Based on the tip type (I, II, or III), it is possible to correlate the best surgical maneuver presented by the authors (Table 2).

Access to the tip is performed through incisions made according to the reduction rhinoplasty technique for the Caucasian nose described by Converse-Diamond (1) (Figures 4 and 5), which means making septocolumellar and intercartilaginous bilateral incisions. In cases that require weakening or cephalic removal of the lower lateral cartilage, the access is performed through an intercartilaginous incision with eversion of the lower lateral cartilage (McIndoe technique) (Figure 6). This technique is carried out preferably with local anesthesia (1:100,000 xylocaine and epinephrine) and sedation. At the end of the surgery, a patch is placed over the nose with a mold (Aquaplast ${ }^{\circledR}$ ) and removed after the seventh day. Other than massages, no other nasal treatment is carried out. 


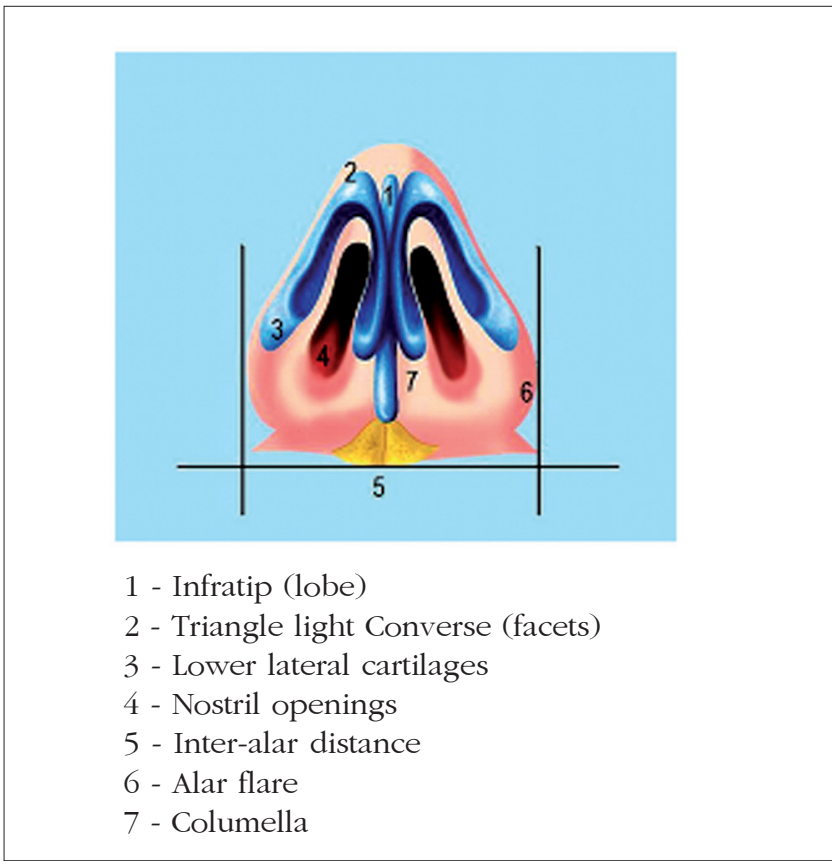

Figure 1. Anatomy and aesthetic points.
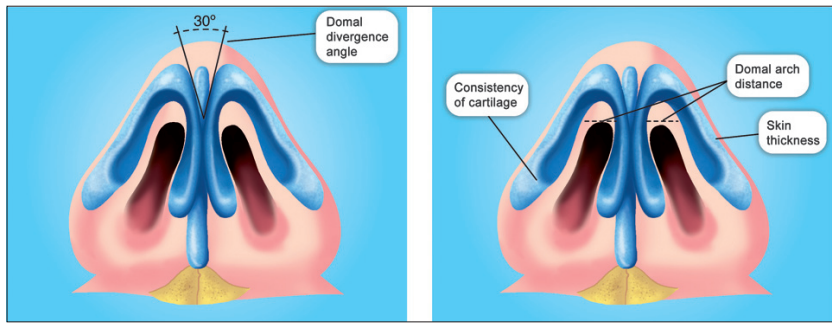

Figures 2 and 3. Points to be analyzed to define nasal tip type.

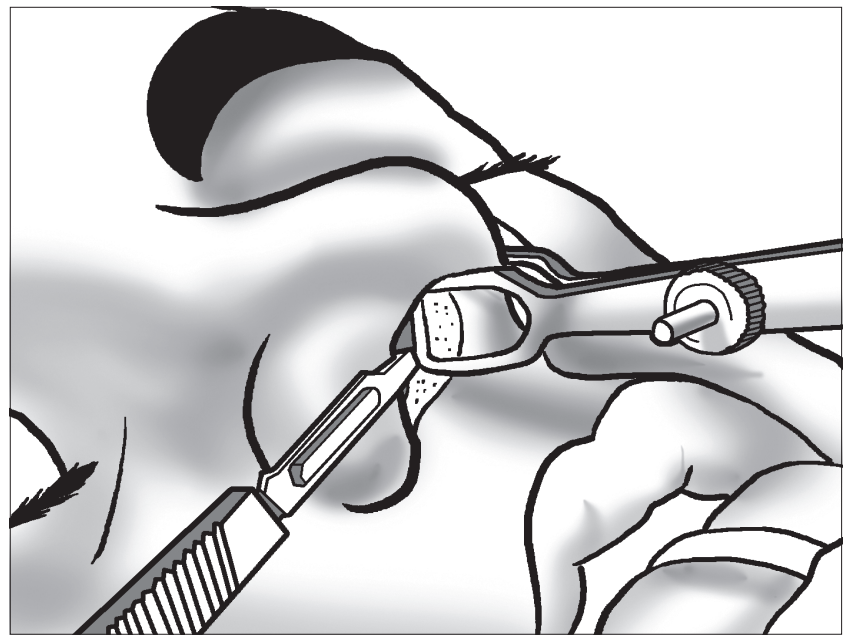

Figure 4. Septocolumellar incision.

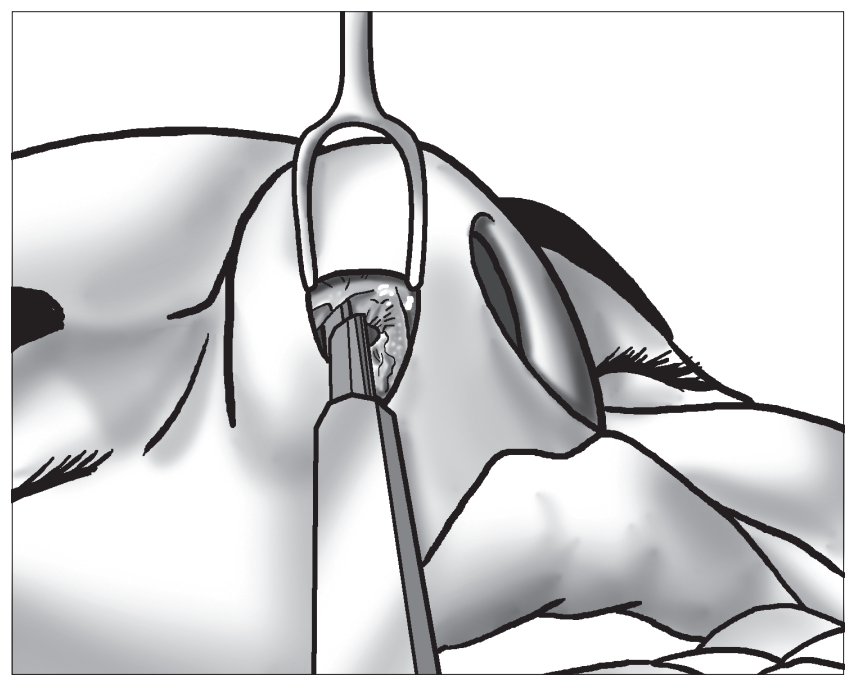

Figure 5. Bilateral intercartilaginous incision.

Table I. Nose tip type classification.

\begin{tabular}{|c|c|c|c|c|c|}
\hline \multirow[t]{2}{*}{ Classification } & \multicolumn{2}{|c|}{ Interdomal Distance } & \multirow[t]{2}{*}{ DA*width } & \multirow[t]{2}{*}{ LLC consistency*** } & \multirow[t]{2}{*}{ Skintype } \\
\hline & DD**** angle & IIC****** distance & & & \\
\hline | & Less than $30^{\circ}$ & Inadequate & Less than 2-3 mm & Weak & Thin \\
\hline$\|$ & Less than $30^{\circ}$ & Inadequate & More than $3 \mathrm{~mm}$ & Moderate & Intermediate \\
\hline III & Less than $30^{\circ}$ & Inadequate & More than $3 \mathrm{~mm}$ & Strong & Thick \\
\hline
\end{tabular}

* Domalarch

** Lower lateral cartilage

**** Domal divergence

****** Intermediate intercrural

Table 2 . Surgical algorithm for nasal tip refinement.

\begin{tabular}{cl}
\hline Surgical maneuvertype \\
\hline I & Interdomal suture \\
II & Interdomal suture with LLC weakening \\
III & Interdomal suture with LLC cephalic removal
\end{tabular}


Surgical technique (Converse-Diamond)steps:

1. Septocolumellarand intercartilaginous bilateral incisions, displacement of the soft tissues from the osteocartilaginous structure.

2. Separation of the upper lateral cartilage from the nasal septum junction, septoplasty with or without turbinate surgery, and reduction of the upper lateral and septal cartilage.

3. Nasal tip refinement based on the proposed surgery algorithm*.

4. Bone hump reduction using cutting-type Maury-Parkes.

5. Osteotomies side by pecking performed with an osteotome Converse $3 \mathrm{~mm}$ in women and $4 \mathrm{~mm}$ in men. Fracture and digital compression.

6. Septocolumellarand intercartilaginous incisions suture. *Displacement of the skin from the nasal tip is carried out (La Garde maneuver).

\section{Interdomal suture technique}

The interdomal suture must be performed on the lower lateral cartilage dome. Its tightening must be gradual and progressive, permitting adjustments related to the definition of a more suitable tip. It must be carried out after septocolumellar and intercartilaginous suture using Monocryl ${ }^{\circledR}$ absorbable thread or colorless PDS ${ }^{\circledR} 4.0$ thread with a straight, sharp needle. Performing the interdomal suture at the end of the surgery prevents excessive manipulation of the nose tip and possible enlargement of the interdomal distance.

Interdomal suture steps (Figures 7-12).

1. Subsequent to the lower lateral cartilage skin displacement using La Garde's maneuver, introduce the $\mathrm{PDS}^{\circledR} 4.0$ thread to the inner portion of the dome. The thread must be introduced from right to left at the posterior border, returning from left to right to the anterior border, more cephalic.

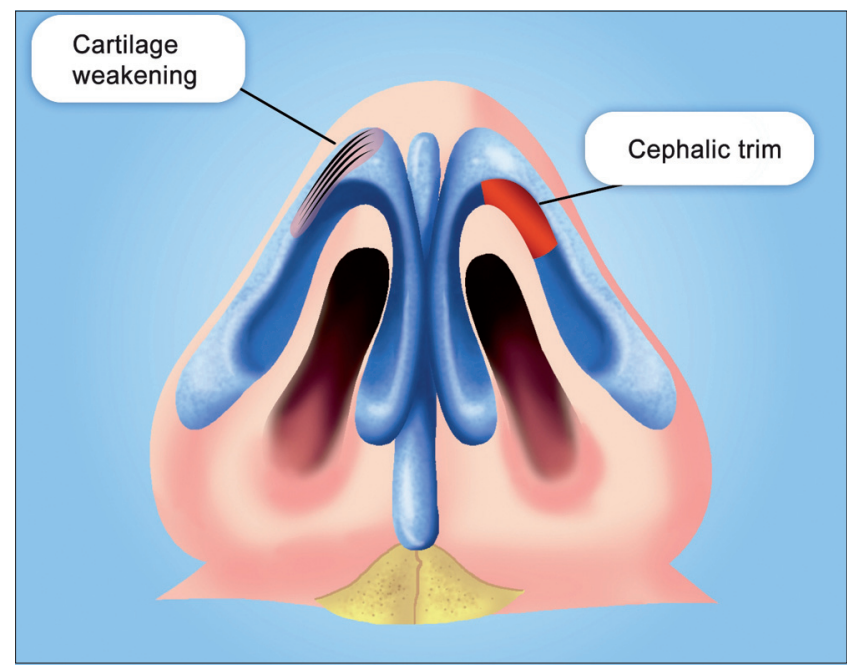

Figure 6. LLC weakening and/or cephalic removal.
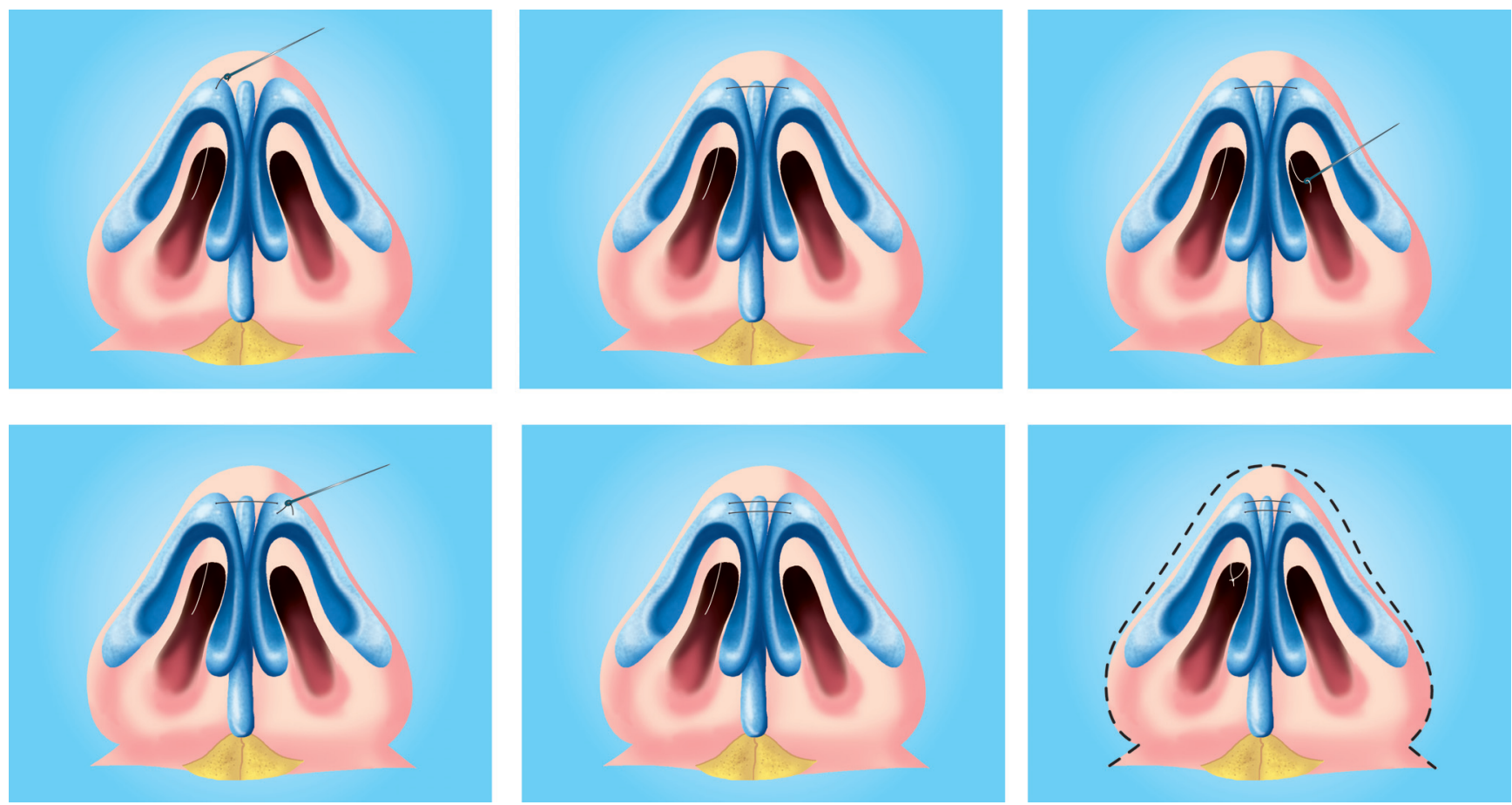

Figures 7 a 12. Subsequent to the lower lateral cartilage skin displacement using Le Garde's maneuver, introduce the PDS ${ }^{\circledR} 4.0$ thread to the inner portion of the dome. The thread must be introduced from right to left at the posterior border, returning from left to right to the anterior border, more cephalic. Return the needle to the nostril in which the suture was initiated, tightening the suture gradually.. 
2. Return the needle to the nostril in which the suture was initiated, tightening the suture gradually.

\section{DISCUSSION}

The substitution of cartilaginous resection techniques by suture techniques to improve nasal tip definition is a global trend (5).

The final result of the suture is influenced mainly by factors such as the cartilage intrinsic force, degree of suture tightening, and the limitation imposed by soft tissues (ligaments, subcutaneous tissue, and skin).

Skin thickness is a determining factor in the effectiveness of the interdomal suture. Results are more significant in patients with thin skin and sparse subcutaneous tissue. However, the interdomal suture may be ineffective in patients with thicker skin and excessive subcutaneous tissue, requiring a reduction of the angle of domal divergence and of the lateral intercrural width, domal arch width, and domal definition through other techniques such as transdomal suture (delivery), or alar lateral spanningtype suture, lateral intercrural suture (6), as well as the Goldmantechnique.

Some details concerning the interdomal suture must be observed:

1. Slight projection of the supratip region (2). The degree of tip definition depends on where the suture is placed. The more lateral the suture, the better the nasal tip projections and definition.

2. A suture more to the anterior may result in slight pinching of the skin and caudal rotation.

3. Discrete pinching of the supra-alar region, especially in patients with very thin skin and weak supra-alar cartilage, when performed at a distance greater than $2-3 \mathrm{~mm}$ from the nasal dome.

4. Asymmetry of the tip can be observed if the sutures are not placed at corresponding levels.

5. Secondary pollybeak.

The suture can be held using long-term absorbable threads or with nonabsorbable threads considering the tensile strength and absorption levels of each material. We currently use Monocryl 4.0 or PDS 4.0 absorbable thread (2).

\section{CONCLUSION}

The interdomal suture is an effective method to improve nasal tip definition on the Caucasian nose and can be performed through endonasal rhinoplasty without delivery. Such a technique has already been reported in the

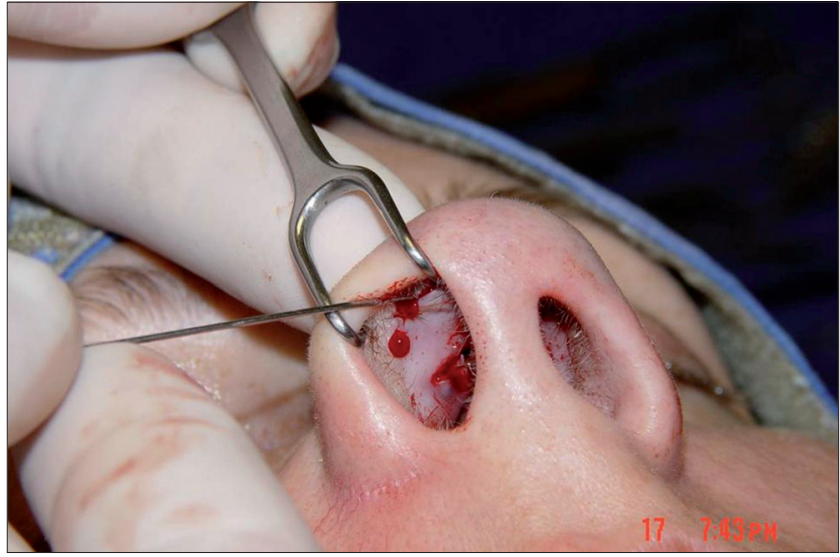

Figure 13. Step 1. Exposure of the domal area with a straight, sharp needle and thread.

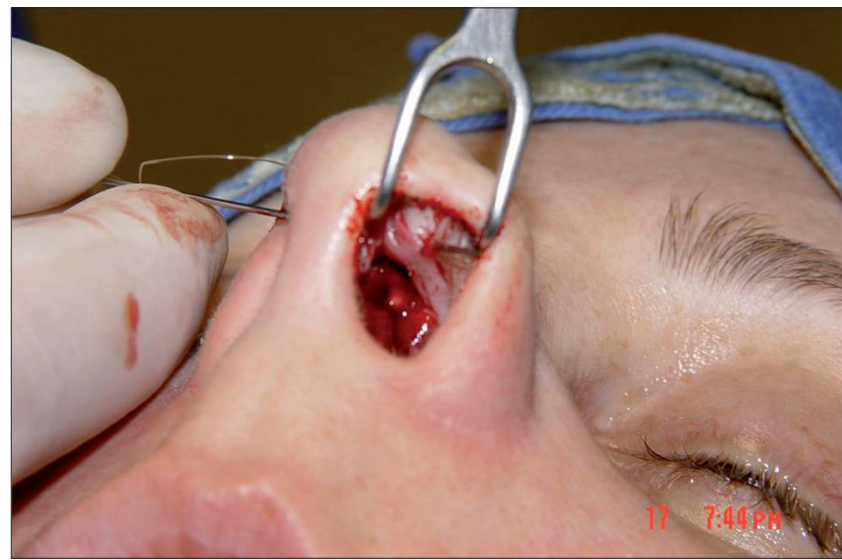

Figure 14. Step 2. Exposure of the intralateral domal area through which the needle and thread will pass.

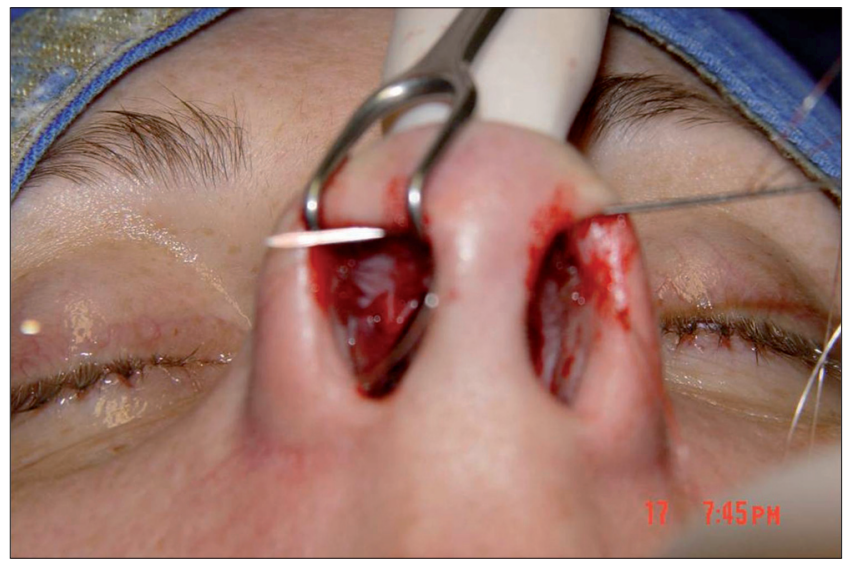

Figure 15. Step 3. Returning the needle through the more interior portion of the domal area. 
literature through open and closed access with delivery, but not through closed access with intercartilaginous and septocolumellar incisions for surgery on the Caucasian nose.

This suture is easy to perform, predictable, controlled, and extremely useful to the surgeon dedicated to nasal tip surgery.

The main sutures applied on the nasal tip were reported by GUYURON and BEHMAND in 2003 (6). Our use of the interdomal suture has produced admirable results for more than 13 years. Our records show that only one patient presented complications: recurrent cellulites of the tip, which resolved after suture removal.

\section{REFERENCES}

1. Maniglia AJ, Maniglia JJ, Maniglia JV. Rinoplastia EstéticaFuncional-Reconstrutora. 1a ed. Revinter 2002:129-150.

2. Corrado A, Bloom J, Becker D. Domal Stabilization Suture in Tip Rhinoplasty. Arch Facial Plast Surg, 2009;11(3):194-7.

3. Tardy ME, Brown RJ. Surgical anatomy of the nose. New York: Raven Press, 1990.
4. Rohrich RJ, Adams WP. The Boxy Nasal Tip: Classification and Management Based on Alar Cartilage Suturing Techniques. Plast Reconst Surg, 2001; 1849:107

5. Tebbetts JB. Discussion:nasal tip sutures part I: the evolution. Plast Reconst Surg, 2003; 12(8):1146-9.

6. Guyuron B, Behmand R. Nasal tip sutures part II: the interplays. Plast Reconst Surg, 2003; 12(8):1146-9.

7. LoS, Rowe-Jones J. Suture techniques in nasal tip sculpture: current concepts. J Laryngol Otol, 2007; 121(8):e10.

8. Gruber R, Weintraub J, pomerantz J. Sutura Techniques for Nasal Tip. Aesthetic Surg J, 2008; 28:92-100.

9. Perkins S, Patel A. Endonasal Suture Techniques in Tip Rhinoplasty. Facial plast Surg Clin North Am, 2009; 17(1):4154.

10. Leach JL, Athré. Four suture tip rhinoplasty: A powerful tool for controlling tip dynamics. Otol Head Neck Surg, 2006; 135:227-31. 